\title{
Nanocomposite Fibre Fabrication via in situ Monomer Grafting and Bonding on Laser-generated Nanoparticles
}

\author{
D. D. van 't Zand ${ }^{1}$, P. Nachev ${ }^{2}$, R. Rosenfeld ${ }^{3}$, P. Wagener ${ }^{1}$, A. Pich ${ }^{2}$, D. Klee ${ }^{2}$, S. Barcikowski ${ }^{1}$ \\ ${ }^{I}$ Technical Chemistry I and Center for Nanointegration Duisburg-Essen (CeNIDE), University \\ of Duisburg-Essen, Universitaetsstr. 5-7, \\ D-45141 Essen, Germany \\ E-mail: stephan.barcikowski@uni-due.de \\ ${ }^{2}$ Interactive Materials Research, DWI an der RWTH Aachen e. V., RWTH Aachen University, \\ Forckenberg Str. 50, D-52056 Aachen, Germany \\ ${ }^{3}$ Laser Zentrum Hannover e.V., Hollerithallee 8, 30419 Hannover, Germany
}

\begin{abstract}
Bioactive nanocomposites may become an important material if both the carrier matrix and the nanoparticle are biocompatible, like is known for zinc oxide and lactones. The fabrication of such nanocomposite made of polycaprolactone nanofibres with embedded nanoparticles is studied during laser ablation in liquid monomer and polymer solution. The in situ conjugation of zinc oxide nanoparticles with $\varepsilon$-caprolactone followed by zinc-initiated polymerization was studied. Indication for covalent bonding between the zinc oxide nanoparticles and the carboxylic units of the oligomers is observed. In addition to the study of the intended nanohybrid formation, possible formation of unintended byproducts was investigated. Laser-induced pyrolysis of solvent was studied for nanosecond, picosecond, and femtosecond laser pulse durations at the same energy input, where all pulse durations caused unintended solvent modification and picosecond pulses were most efficient for nanoparticle production. Heading towards fabrication of macroscopic bioactive fibre pads, the lasergenerated zinc oxide polymer nanocomposite have been successfully spun into nanofibres using electrospinning. Polymer-embedding is demonstrated at the example of macroscopic nanocomposite fibre pads with various bio-relevant nanoparticles fabricated by laser ablation of magnesium, iron, and tantalum in polycaprolactone solution.

DOI:10.2961/jlmn.2012.01.0004
\end{abstract}

Keywords: $\varepsilon$-caprolactone, zinc oxide, monomer grafting, pulsed laser ablation in liquid

\section{Introduction}

Biocompatible and biodegradable polymeric materials are of great interest for creating biomaterials with applications in the medical field [1], where supported wound healing is under intense study [2]. The biological function and regeneration of dermal tissue depends on an adequate supply of not only nutrients, but also trace elements [3]. Amongst other trace elements, the presence of zinc ions is an important factor in the process of wound healing, and it is of medical interest to think of ways to gently feed the skin with these ions [4]. For supplying (damaged) skin tissue with trace elements, it is desirable to embed nanoparticles (NPs) made of zinc oxide $(\mathrm{ZnO})$ in a wound dressing so that ions can be released which in turns aids faster healing of the wound [5]. This is where the use of biocompatible polymeric materials comes into play. Not only the chosen polymer needs to be e.g., non-toxic and highly porous, but it is also crucial that the NPs are fixed within it avoiding particle migration so that the NPs are prohibited from contacting with the skin and possibly entering the body via the wound. An appealing polymeric matrix of medical relevance that may carry and safely embed metallic NPs from which ions can be released is $\varepsilon$-polycaprolactone (PCL) [6]. PCL is suitable for controlled drug delivery since it is highly permeable to many drugs and has excellent biocompatibility [6]. For making optimum use of properties of such a NP polymer composite, having a large surface area is of profound importance for the composites' activity. Electrospinning is an exquisite way of achieving this [7]. In this technique, the polymer (which may contain NPs) is spun into very small fibres, thus creating a large surface area. A nice feature is that the electrospun material can be easily applied to a wide variety of surfaces, including dermal tissue and wound dressings $[8,9]$. The main topic of this paper is the entire process chain for creating an electrospun $\mathrm{ZnO}$ PCL nanocomposite for a biomedical application. Fundamental steps for creating such a composite are the NP synthesis, incorporating these into the monomer, and finally the polymerization of $\varepsilon$-caprolactone $(\mathrm{CL})$ into $\mathrm{PCL}$, which then embodies the $\mathrm{ZnO}$ NPs. The $\mathrm{ZnO}$ initiates the polymerization of CL, a highly desirable feature in our case, since the ultimate aim is to embed the $\mathrm{ZnO}$ NPs in PCL compatible to electrospinning. Polymerization of CL into PCL in presence of zinc can be realized via raising the temperature or use a catalyst at ambient temperature. The $\mathrm{ZnO}$ NPs used in our investigation are created via laser ablation on $\mathrm{Zn}$ in liquid [10,11], a method that is of growing importance in the field of synthesis and shaping of colloidal nanoparticles $[12,13,14]$. It recently has been shown that incorporation of laser-ablated NPs in situ is possible by solvent casting [15]. For successful incorporation and homogeneous particle dispersion into a polymeric matrix it is crucial that the NPs can be functionalized with polymer molecules or other ligand molecules. Pulsed laser ablation 
in liquid (PLAL) is an extremely effective synthesis method allowing in situ grafting of ligand molecules on lasergenerated colloidal NPs $[15,16,17,18]$. Under the perspective of biomaterial application, it is of particular interest to determine and understand potential damage to the molecules caused by laser irradiation since pyrolysis of the solvent can affect the stability and surface accessibility of the nanoparticle surface [19]. Recently it was demonstrated that the laser ablation of metallic NPs in organic solvents is accompanied by pyrolysis of the solvent of which the product subsequently interacts with the metal NPs to form e.g. a graphitic layer onto the surface $[20,21]$. Besner et al. observed that laser irradiation of a (plasmon resonant) metal nanoparticle colloid may result in the pyrolysis of polymeric media to some extent via oxidative processes [22]. On the other hand, the use of ultrashort pulses was reported to minimize thermal impact on the material [23]. Nevertheless, an investigation of laser-induced pyrolysis applying different pulse durations (from ultrafast to nanosecond pulse regime) under comparable conditions (using the energy input to the liquid) is still missing.

This paper is organized following the zinc nanoparticle nanocomposites and nanofibre fabrication procedure. In the next section, we describe the experimental procedures including sample preparation. The following section deals with the polymerization study of CL in presence of zinc, the electrospinning results and finally the pyrolysis study of tetrahydrofuran (THF).

Since systematic variation of the whole laser, liquid and polymerisation parameter sets is almost impossible, we present an engineering approach more likely than fundamental physical-chemical studies, driven by the idea to fabricate and engineer an electrospun nanofibre biomaterial based on educts derived from laser ablation in liquids. The result on the monomer grafting on the laser-fabricated nanoparticles, effect of elevated polymerisation temperature, as well as laser pulse duration effects on liquid pyrolysis presented in the following aim to shine light on boundary conditions that have to be considered during engineering of the PLAL methods towards nano-integration into bioactive fibre pads.

\section{Experimental}

PLAL was performed by ablating zinc in tetrahydrofuran (THF) at two different settings. First, at ambient temperature in a mixture of CL/THF with added 2ethylhexanoic acid ( $0.012 \mathrm{vol} \%$, serves as catalyst) using a femtosecond laser to study the monomer grafting in general. Second, at elevated temperature $\left(140^{\circ} \mathrm{C}\right)$ in $\mathrm{CL}$ using a high-power picosecond laser to fabricate sufficient amount of nanocomposite for electrospinning. The higher temperature is necessary to initiate polymerization in a degree that is sufficient for electrospinning. Laser ablation at ambient temperature ends up at oligomers which are not suitable for electrospinning. For this reason the temperature was increased to $70^{\circ}, 80^{\circ}, 120^{\circ}$ and $140^{\circ} \mathrm{C}$. Only at $140^{\circ} \mathrm{C}$ during the ablation of $\mathrm{Zn}$ the polymerization took place in a sufficient way for later electro-spinning. At ambient temperature the laser parameters were: $120 \mathrm{fs}$, wavelength $800 \mathrm{~nm}$, pulse energy $250 \mu \mathrm{J}, 300 \mathrm{sec}$ irradiation time (Spitfire Pro femtosecond laser), and at $140^{\circ} \mathrm{C}: 7 \mathrm{ps}$, wavelength 1030 $\mathrm{nm}$, pulse energy $250 \mu \mathrm{J}$, total ablation time $300 \mathrm{~s}$. The target was irradiated with a spiral scan pattern. The reaction vessel was continuously stirred using a magnetic stirrer to ensure the liquid in front of the target was free from newly formed NPs or bubbles before subsequent irradiation pulses (see $[24,25])$. After the ablation procedure, the degree of polymerization was assessed using gel permeation chromatography (GPC).

The formed $\mathrm{ZnO}$ PCL nanocomposites were spun into fibres using an electrospinning apparatus (Generator: Eltex $\mathrm{KNH} 34 / \mathrm{N} 2 \mathrm{~A}$ operated at $17.5 \mathrm{kV}$ DC, spinneret-target distance $20 \mathrm{~cm}$, syringe with needle, flow rate $0.4 \mathrm{ml} \mathrm{h}^{-1}$, solvents: mixture of THF and DMF (volume ratio 1:1)). The PCL concentration in THF/DMF is $440 \mathrm{~g} \mathrm{l}^{-1}$. To allow fabricating very small fibres, it was necessary to mix the formed nanocomposite with higher molecular weight PCL (mass ratio $\sim 1: 150$ ) while dissolved in THF. After mixing and evaporation of THF we end up with a homogeneously mixed nanocomposite suitable for electrospinning. The electrospun nanocomposites were characterized using TEM (ZEISS Libra 120, $120 \mathrm{kV}$, coating on Formvar grid directly by electrospinning) and scanning electron microscopy (SEM, Hitachi S-3000N, $15 \mathrm{kV}$, the fibres are deposited directly by electrospinning on an aluminium foil substrate after which the sample is sputtered with gold.). Further electrospinning experiments with composites containing different laser-generated NPs (made from PLAL of iron, magnesium, and tantalum) were performed in the same manner, except here the PCL concentration in THF used is $8.9 \mathrm{gl}^{-1}$.

The pyrolysis of THF was investigated comparing varying laser irradiation conditions: (i) $120 \mathrm{fs}$, repetition rate $5 \mathrm{kHz}$, wavelength $800 \mathrm{~nm}$, pulse energy $250 \mu \mathrm{J}$, $1800 \mathrm{~s}$ irradiation time (Spitfire Pro femtosecond laser) (ii) $7 \mathrm{ps}$, repetition rate $5 \mathrm{kHz}$, wavelength $515 \mathrm{~nm}$, pulse energy $125 \mu \mathrm{J}, 3600 \mathrm{~s}$ irradiation time (TruMicro 5250 picosecond laser) (iii) $50 \mathrm{~ns}$, repetition rate $5 \mathrm{kHz}$, wavelength $1064 \mathrm{~nm}$, pulse energy $6.5 \mathrm{~mJ}, 70 \mathrm{~s}$ irradiation time (Rofin RSM 100D nanosecond laser). Since the energy transferred to the sample per unit time is different for each laser parameter set, we fixed the total energy input to be $2250 \mathrm{~J}$ in all pyrolysis experiments to be able to draw an energy input specific comparison of the results. Pyrolysis products were analyzed using FTIR spectroscopy ( $\mathrm{KBr}$ pellet, Thermo Nicolet Nexus 470).

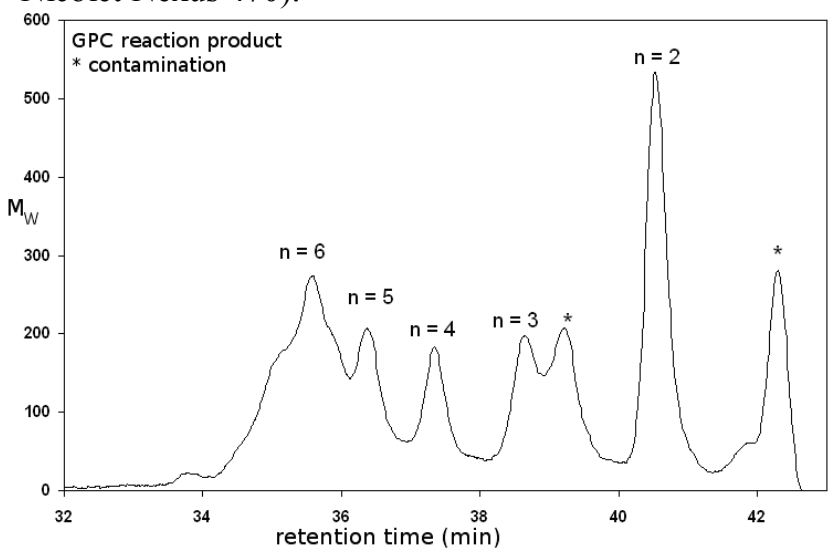

Fig. 1: GPC chromatogram ( $\mathrm{M}_{\mathrm{W}}$ (weight average molecular weight) against retention time) of the reaction product. Oligomers consisting of a higher number of monomers (n) are eluted most quickly. Oligomers ranging from $n=2$ up to $n=6$ are observed. 


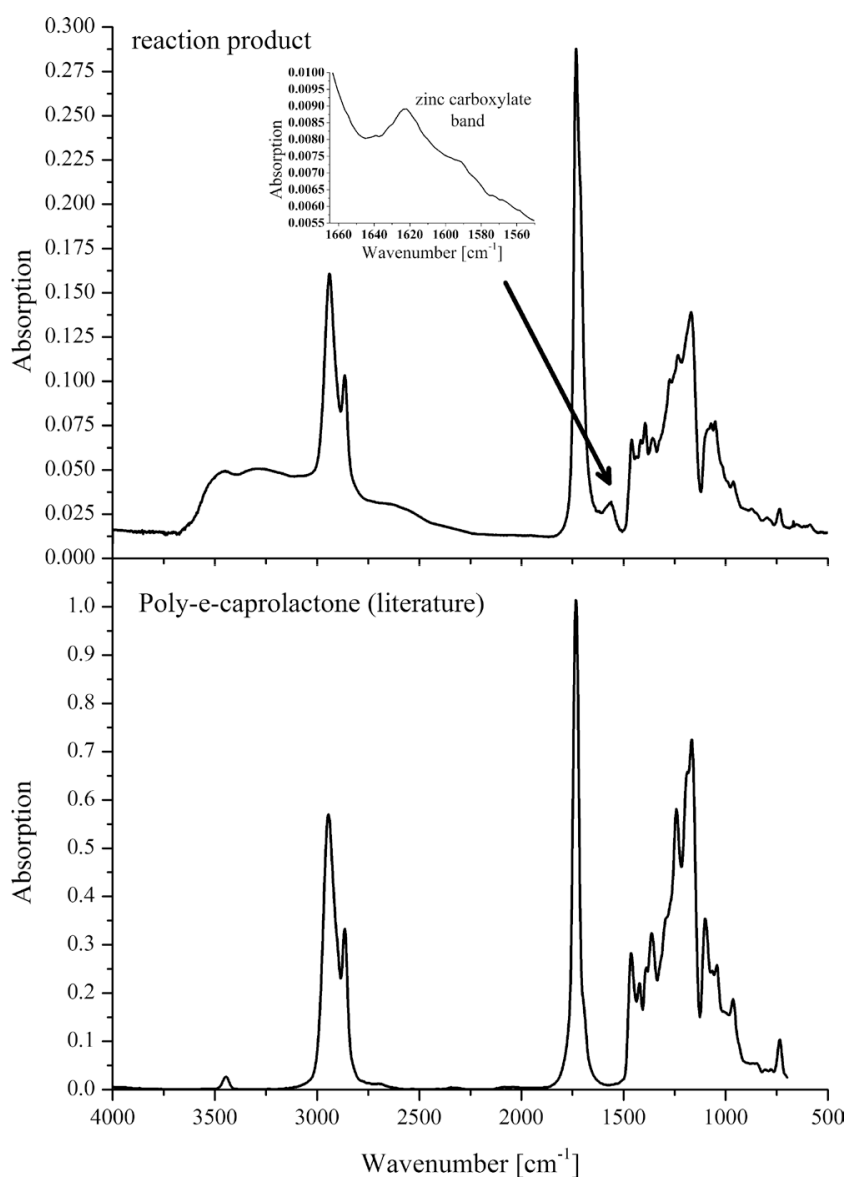

Fig. 2: FTIR spectra of the reaction product (top) and pure $\varepsilon$ polycaprolactone (bottom). The bonding between the zinc and polymer is evidenced by the appearance of the peak at wavenumber $1550 \mathrm{~cm}^{-1}$.

\section{Results and discussion}

In this section first the ablation of zinc foil immersed in CL is studied, with the aim to conjugate the ZnO NPs in situ and polymerize CL into PCL. As we showed in a prior publication, laser ablation zinc yields completely oxidized and crystalline $\mathrm{ZnO}-\mathrm{NP}$ [26]. The NP characterization is presented in section 3.1. The in situ polymerisation is performed in the presence of a catalyst (section 3.2) and at elevated temperature without a catalyst (section 3.3), respectively. Subsequently, PCL with embedded ZnO NPs is used for fabricating nanofibres via electrospinning (section 3.4). Moreover, it is demonstrated that this procedure can be applied to composites containing different types of NPs derived from laser ablation of metals. The extent of damage occurring in organic matter under different laser ablation conditions is investigated in section 3.4.

\subsection{Polymerization using laser-ablated zinc}

Zinc foil is ablated at ambient temperature in presence of CL, THF and the catalyst (2-ethylhexanoic acid, 0.012 vol\%) (120 fs, $800 \mathrm{~nm}, 250 \mu \mathrm{J}, 300 \mathrm{~s}$ irradiation). The fabricated nanocomposite is analyzed using GPC and FTIR spectroscopy. The catalyst was added to the solution to facilitate polymerization without the need to raise the temperature. It is known that $\mathrm{ZnO}$ NPs act as an initiator of the polymerization reaction.

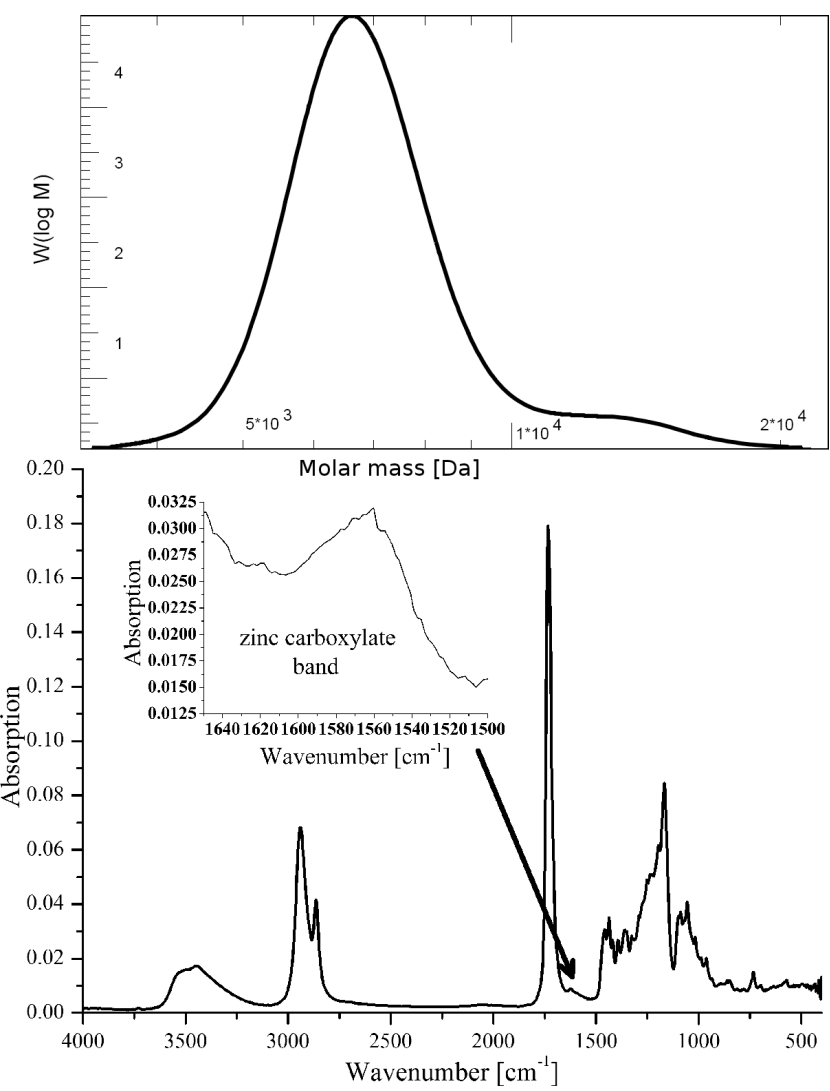

Fig.3: Top: GPC chromatogram of the reaction product showing full polymerization and a narrow distribution in the molar mass. Bottom: FTIR spectrum in which the bonding between the zinc and the $\varepsilon$-polycaprolactone is confirmed again.

In Fig. 1 the resulting GPC chromatogram is shown. From the chromatogram it is obvious that complete polymerization was not achieved, however it is evident that the monomer does polymerize to a certain extent. Oligomers with lengths of up to six monomers $(n=6)$ are detected using GPC (Fig. 1). In Fig. 2, FTIR spectra from the reaction product and pure PCL are shown. When comparing the spectra, an additional small absorption peak at around wavenumber $1550-1600 \mathrm{~cm}^{-1}$ differentiates the spectrum measured for the reaction product from the unmodified polymer. This is an indication that there is interaction, and may be covalent bonding between the surface of the zinc oxide NPs and the carboxylic units of the oligomers [27, 28]. The oligomer is formed by monomer engrafting on the NPs, after which it polymerizes into oligomers of sizes ranging from two till six monomer units.

\subsection{Polymerization at elevated temperature $\left(140^{\circ} \mathrm{C}\right)$}

In the previous section it was found that CL partly polymerizes in the presence of $\mathrm{ZnO}$ and catalyst. The procedure carried out here is analogous to the one presented in the previous section. In order to accomplish polymerization, elevated temperature rather than the presence of a catalyst (2-ethylhexanoic acid, $0.012 \mathrm{vol} \%$ ) in the solution was employed. Zinc foil is immersed in CL which is held at elevated temperature $\left(140^{\circ} \mathrm{C}\right)$ and stirred for 60 minutes to ensure that the polymerization reaction takes place. Ablation of zinc foil was performed for 300 seconds ( 7 ps, 1030 $\mathrm{nm}, 250 \mu \mathrm{J})$, after which the properties of the product were assessed using GPC and FTIR spectroscopy. In Fig. 3 (top) 
the chromatogram displays a single (broad) peak, indicating the formation of polymer, rather than a series of oligomers, as was observed in the results discussed in the previous section. To quantify the degree of polymerization, the polymerization index (PMI) is considered. The PMI value of 1.07 indicates a narrow length distribution of the polymer chains, which means that the polymerization takes places in a controlled manner. For further verification the FTIR spectrum in Fig. 3 is compared to the model spectrum of PCL (Fig. 2 bottom), from which it is clear that PCL has been formed in the ablation procedure. Besides, again a small peak around wavenumber $1550-1630 \mathrm{~cm}^{-1}$ is visible. This is the peak that is associated with the interaction between hydroxyl groups from the zinc surface and carboxylate groups from the polymer [29, 30, 26, 17]. The position of the zinc carboxylate bands in the two FTIR spectra in the previous and this section are marginally different. Most likely this is caused by the somewhat chemically different environments, such as e.g. the presence of different amounts of trace water. There are some differences between the two spectra of the composite, mostly around 2700-3700 $\mathrm{cm}^{-1}$. The broad peak around $3600 \mathrm{~cm}^{-1}$ is consistent with $-\mathrm{OH}$ groups, indicating the presence of water. This peak broadening is less prominent in the spectrum taken from the composite created at elevated temperature (Fig. 3), indicating there is less water present compared to the composite created at room temperature (Fig. 2). Importantly, when comparing the literature spectrum with both experimental spectra (Figs. 2 and 3), there is barely evidence of damage to PCL after laser ablation which could potentially result in pyrolysis of PCL. This effect is more prominent in presence of THF during laser ablation. Consequently, laser-induced pyrolysis of the solvent is an important issue during the laser-based fabrication of nanocomposites and will be discussed in section 3.4.

In both polymerization investigations, the one involving a catalyst and the one performed at elevated temperature, it is found that interaction between the ZnO NPs and the monomer takes place. Already at relatively short irradiation times $(300 \mathrm{sec})$ successful conjugation of the monomer units on the NPs is realized. Especially at elevated temperature, relatively high degree of polymerization and narrow molecule size distribution has been obtained. This suggests that the use of laser ablation is an effective method for the chemical in situ grafting of monomer molecules on zinc NPs.

\subsection{Electrospinning}

The nanocomposite synthesized by laser ablation using in situ generation and conjugation of NPs in PCL are used for electrospinning experiments for making nanofibres. To
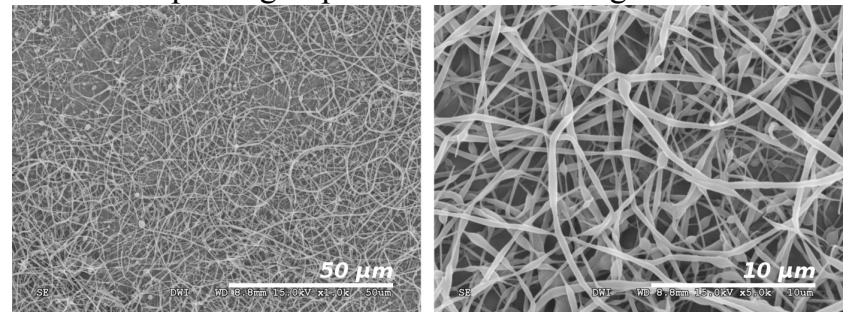

Fig. 4: SEM micrographs taken from the nanocomposite after electrospinning. The quality of the fibres is evidenced by the small number of beads appearing in the fibres.

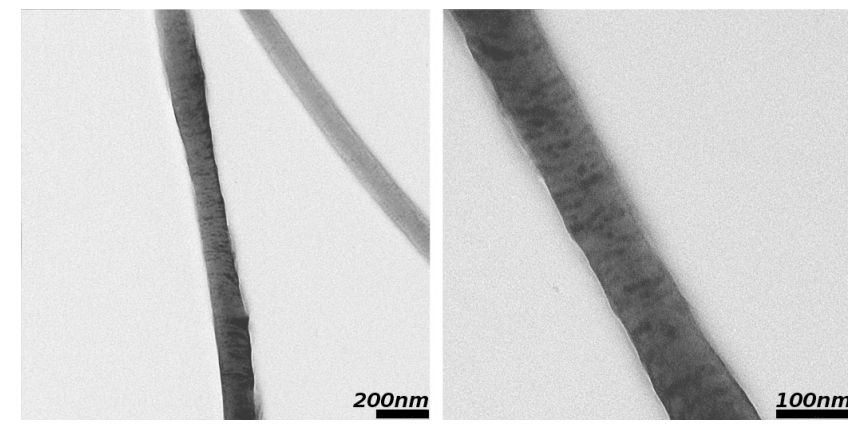

Fig. 5: TEM micrographs taken from the nanocomposite after the electrospinning procedure.

succeed in making decent fibres using electrospinning it is crucial that the molar weight of the polymer is sufficiently high. In case the molar weight of the nanocomposite is not sufficiently high, it can easily be mixed with PCL that has a higher molar weight, as described in the experimental section. After mixing with higher molecular weight PCL the nanocomposite is electrospun successfully into nanofibres with diameters ranging from $\sim 200-500 \mathrm{~nm}$, see Fig.4. The micrograph on the right shows the fibres in more detail (scale bar $10 \mu \mathrm{m}$ ). In the SEM micrographs the fibres deposited on the substrate are clearly visible. The fibres show little imperfections, evidenced by the small number of beads appearing in the fibre structure. The successful electrospinning result implies the potential for preparing nonwoven nanofibres with a high specific surface. To investigate the electrospun nanocomposite in more detail, TEM micrographs were taken (Fig. 5). In these micrographs the ultrastructure of the fibres is made visible. The $\mathrm{ZnO}$ NPs are homogeneously distributed within the fibres, ensuring functionality throughout the entire composite.

The applicability of this method to different materials is of high interest as well. Therefore, we performed electrospinning experiments on nanocomposites loaded with dif-

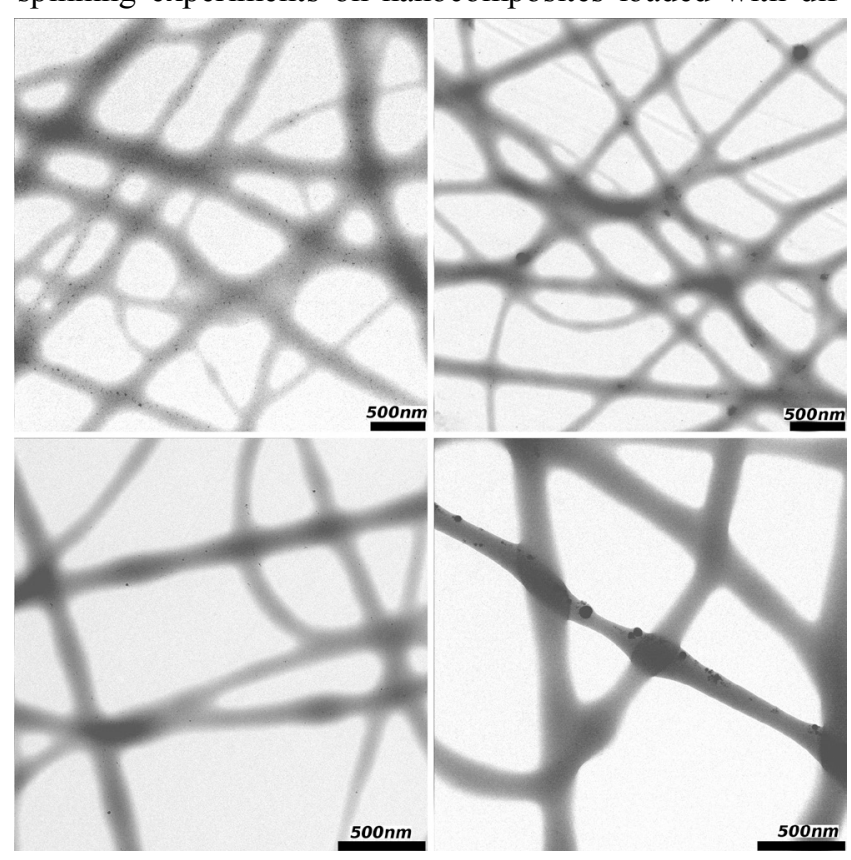

Fig. 6: TEM micrographs taken from nanocomposites spun into nanofibres using electrospinning. The nanocomposites contain zinc (top left), magnesium (top right), iron (bottom left), and tantalum (bottom right). 
ferent NPs derived from laser ablation of the respective metals in PCL solution. The chosen materials apart from zinc are magnesium, iron, and tantalum, since these materials are attractive components of nanocomposites intended for biomedical applications [3, 4]. Magnesium is known to be an important factor in cell growth [31], iron NPs are widely used for numerous in vivo applications such as magnetothermia [32], and tantalum is attractive for the use as a bio-inert contrast agent in x-ray imaging [33]. Before the electrospinning procedure the respective metal targets were laser-ablated when immersed in commercial PCL at elevated temperature $\left(140^{\circ} \mathrm{C}\right)$. In Fig. 6 micrographs are shown of the nanofibres fabricated out of these nanocomposites using electrospinning. The NPs are essentially homogeneously dispersed within the different nanocomposites, which allows one to utilize the properties of the NPs fully.

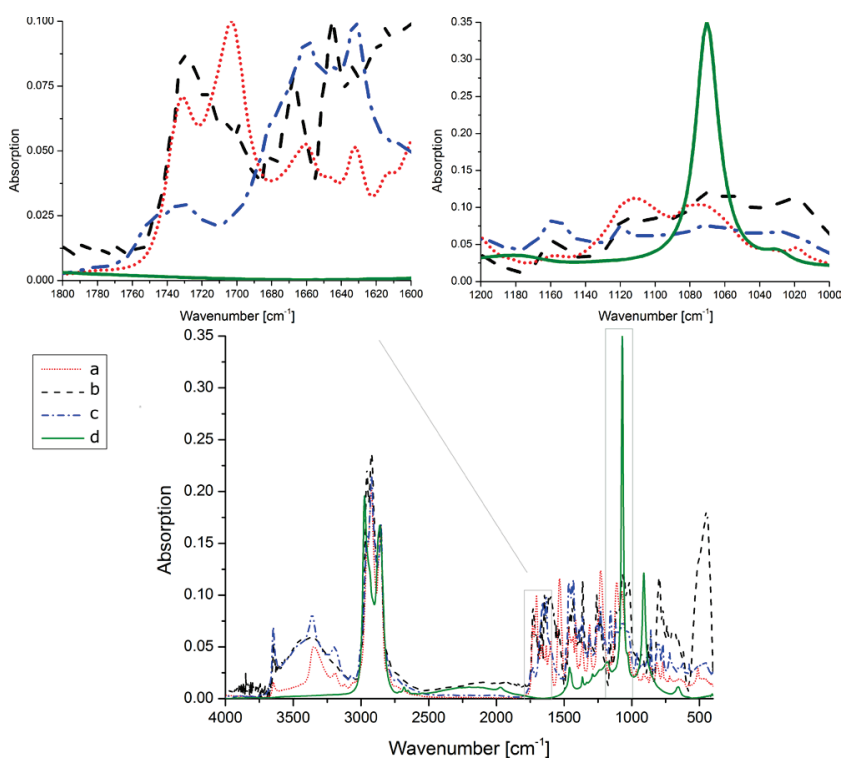

Fig. 7: FTIR spectra of THF treated under varying laser conditions: (a) nanosecond (b) picosecond, (c) femtosecond, and (d) untreated THF.

\subsection{Pyrolysis of THF}

It is investigated to what extent potential pyrolysis of the solvent THF occurs depending on the laser ablation conditions. To be able to distinguish the effect of PLAL clearly, pure THF is used in absence of any other organic molecules.). The scope of this paper is to investigate the whole process chain starting from the fundamental aspects to fabrication of an applicable bioactive fibre. This includes the right choice of the laser source not only in terms of productivity but also considering pyrolysis and subsequent production efficiency. Consequently, we investigated the pyrolysis effects of different pulse durations ranging from femto- to nanosecond pulses under comparable conditions. For this purpose, the total laser energy applied to ablate the sample is held constant at $2250 \mathrm{~J}$, using a femtosecond, picosecond, and nanosecond laser, respectively. The product is analyzed using FTIR spectroscopy. In Fig. 7 the FTIR spectra of the ablated $\mathrm{ZnO}$ (lines $a-c$ ) in presence of THF are shown along with the spectrum measured for untreated THF (line $d$ ). A clear observation for all three ablation experiments is that THF is modified during the abla- tion procedure. For better comparison, all spectra are normalized with respect to the peak at $2850 \mathrm{~cm}^{-1}$ which is related to $\mathrm{CH}_{2}$ groups. However, a possible contamination with water or residual polymer that resisted the cleaning procedure may introduce some inaccuracies. In matter of the pyrolysis of THF during this process areas of 1600 $1800 \mathrm{~cm}^{-1}$ and $1000-1200 \mathrm{~cm}^{-1}$ are enlarged. At $1070 \mathrm{~cm}^{-1}$ one can see the dominant C-O-C peak of THF, which decreases significantly after laser ablation. This indicates that these bonds are extensively broken. Absorption bands originating from $\mathrm{C}-\mathrm{H}$ and $\mathrm{C}-\mathrm{C}$ bonds (around wavenumber $3000 \mathrm{~cm}^{-1}$ and below $1500 \mathrm{~cm}^{-1}$ respectively) are present before and after ablation treatment. An important change in absorption is found at wavenumbers $1650 \mathrm{~cm}^{-1}$ and $1725 \mathrm{~cm}^{-1}$, which represents olefinic species and species containing carbonyl groups, respectively. This indicates formation of pyrolysis products, possibly partly resulting from ring cleavage induced by the laser ablation [34] When looking more closely at the absorbance around $1650 \mathrm{~cm}^{-1}$, it is clear that the structure of the product is dependent on the laser pulse duration. For the shorter pulse durations (ps lines $b$ and fs lines $c$ ), slightly more olefinic compounds are formed, while ns pulse durations (line $a$ ) create less olefin pyrolysis products. This indicates that for ns pulse duration the solvent is not modified in a way that causes many $\mathrm{C}=\mathrm{C}$ bonds to form during ablation of a solid target in THF. When considering the peak around wavenumber $1725 \mathrm{~cm}^{-1}$ however, a different trend is observed. This carbonyl peak intensity changes when the laser pulse duration is increased; for shorter pulse duration the intensity of the carbonyl peak decreases. The decreasing abundance of carbonyl group with decreasing laser pulse length indicates that the pyrolysis mechanism depends on the laser intensity. Carbonylic species are most likely formed via, e.g., ring cleavage during laser ablation, while at shorter pulse durations (lines $b$ and $c$ ) the appearance of olefinic species becomes more prominent [34].

Summarizing pyrolysis results, it has become clear that species responsible for the absorption peaks around wavenumbers $1650 \mathrm{~cm}^{-1}$ and $1725 \mathrm{~cm}^{-1}$ are influenced to various extents during laser ablation in THF. When performing PLAL experiments, this effect needs to be taken into consideration, especially when one wishes to create products that require biocompatibility since this property may be affected, as well as colloidal NP stability. It was demonstrated by Amendola et al. that using different solvent leads to the formation of NPs and varying pyrolysis byproducts [21]. Nevertheless, as the total process chain is in our interest, the choice of the solvent was done by consideration of many aspects like its ability to dissolve polymer and monomer, useability for electrospinning, resistance against pyrolysis, its hazard potential and finally the price. In total, THF was the most suitable solvent to meet all this criteria. However, the pyrolysis products do not necessarily affect the biocompatible properties to a marked extent, since the solubility of the olefinic compounds often is low and pyrolysis products have high molecular weight. In general carbonylic compounds (dominantly generated during ns laser ablation) are more water soluble compared to olefinic species (dominantly generated during fs and ps laser ablation). Up to now, we have not observed any toxic effects of the laser-generated nanocomposites. But if biomedical ap- 

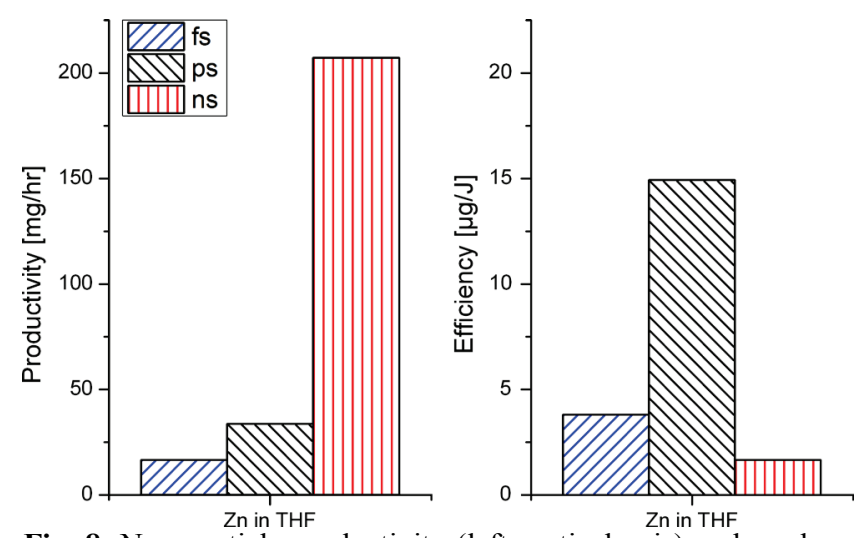

Fig. 8: Nanoparticle productivity (left vertical axis) and production efficiency (right vertical axis) at different pulse durations (PLAL of $\mathrm{Zn}$ in THF).

plication is envisaged, biocompatibility studies (in our case with fibroblast and keratinocytes) have to be carried out to find out if the observed pyrolysis products bear the risk to cause adverse effects during application. Although the solvent pyrolysis products could adhere to the colloidal nanoparticles, it has been found that NiTi nanoparticle coatings generated by fs laser ablation in acetone are fully biocompatible (without purification before testing), and no adverse effects have been found during stem cell growth [35].

Contra-intuitively, shorter pulse duration may not guarantee minimal impact on the liquid matrix at the same laser energy input. Since pyrolysis does not allow favouring a certain pulse duration regime, productivity and efficiency aspects of nanoparticle fabrication may be considered now for all three pulse duration regimes if up-scaling is desired. Productivity of the laser systems is defined as mass ablated from the target and dispersed in the liquid at constant time interval of $\mathrm{fs}$, ps and ns laser ablation of $\mathrm{Zn}$ in THF. Using typical pulse energy regimes (and average power) of industrial lasers available today, it turns out that nanosecond laser ablation is most productive (Fig. 8 left). This result is expected, because of the high average laser power of ns laser system. Production efficiency has additionally been addressed in terms of cumulative laser pulse energy required per mass nanoparticles generated during laser ablation (Fig. 8 right). For energy-specific nanoparticle productivity, it is obvious that ps laser ablation is superior to shorter or longer pulse duration. When employing a femtosecond pulse duration rather than picosecond, the laser fluence may be affected by nonlinear focusing effects, which is stronger at shorter pulse durations [36]. This can explain the higher efficiency observed when picosecond pulse duration is used compared to femtosecond pulse duration.

Hence, for a desired mass of $\mathrm{Zn}$ nanoparticles to be harvested by pulsed laser ablation in THF, ps laser ablation may be an advantageous pulse duration regime. Under this aspect, it is interesting to keep in mind that at the same energy, ps laser ablation does not create pyrolysis products to a higher extent compared to fs or ns laser ablation (at least under our experimental conditions).

\section{Conclusion}

Monomer conjugation to laser-generated nanoparticles has been shown, including the subsequent integration into electrospun macroscopic polymer fibre pads. The grafting is characterized by bonding between the carboxylic units of the monomer or polymer and the surface of the $\mathrm{ZnO}$ nanoparticles. This demonstrates that during laser ablation the monomer is linked to the NP surface in situ, a finding which opens up a vast range of possibility concerning the in situ functionalization of nanoparticles and their embedding into functional macroscopic devices.

The laser ablation of zinc foil immersed in the monomer caprolactone gives best results in terms of polymerization if laser ablation is performed at elevated temperature, where the polymerization proves to be profoundly controlled and the final polymer has a narrow chain length distribution. At ambient temperature, oligomers lengths of two to six monomer units were obtained.

It is demonstrated that the possibility of damage to organic liquid in which laser ablation is carried out must be taken into account, as is evident from our results. Pyrolysis effects are identified during fs, ps, and ns laser ablation in THF. For shorter pulse durations (fs/ps) slightly more olefinic compounds are formed, while at longer pulse duration (ns) more carbonylic species were found. These results initiate an insight in pyrolysis occurring depending on laser intensity. But at the same cumulative laser energy applied for nanoparticle synthesis, all pulse durations caused unintended modification of the liquid. It may be of relevance for future scale-up that, at least under our experimental conditions, ps laser ablation was most energy efficient for nanoparticle fabrication.

After up-scaling, the laser-fabricated $\mathrm{ZnO}$ nanoparticle polycaprolactone nanocomposites enable electrospinning of fibres with nanoparticles made from $\mathrm{Zn}, \mathrm{Mg}, \mathrm{Fe}$, and $\mathrm{Ta}$ homogeneously distributed within the fibre ultrastructure and throughout the macroscopic composite. Hence, this method may be a fruitful procedure for creating macroscopic polymer nanoparticle composite pads with a high specific surface, a property desirable for biomedical application.

Although biocompatibility of laser-generated nanoparticles fabricated in organic solvent already has been shown [35], future investigation aiming at biomedical application will have to include control samples to test if the observed pyrolysis products.

\section{Acknowledgments}

This work was supported by the German Research Foundation (DFG) research program SPP1327.

\section{References}

[1] J. Jagur-Grodzinski. Polym. Advan. Technol., 17:395418, 2006.

[2] S. Guo and L.A. DiPietro. J. Dent. Res., 89:219-229, 2010.

[3] J.A. Liyanage, I. Rodrigo, and M. Wijeratne. Chem. Spec. Bioavail., 18:57-60, 2006.

[4] I. Tenaud, I. Sainte-Marie, O. Jumbou, P. Litoux, and B. Dreno. Br. J. Dermatol., 140:26-34, 1999.

[5] D.K. Heyland, N. Jones, N.Z. Cvijanovich, and H. Wong. J. Parenter. Enteral. Nutr., 32:509-519, 2008.

[6] M.A. Woodruff and D.W. Hutmacher. Prog. Polym. Sci., 35:1217-1256, 2010.

[7] Z.M. Huang, Y.Z. Zhang, M. Kotaki, and S. Ramakrishna. Compos. Sci. Technol., 63:2223-2253, 2003. 
[8] P. Rujitanaroj, N. Pimpha, and P. Supaphol. Polym., 49:4723-4732, 2008.

[9] P. Zahedi, I. Rezaeian, S.O. Ranaei-Siadat, S.H. Jafari, and P. Supaphol. Polym. Advan. Technol., 21:77-95, 2010.

[10]R.K. Thareja and S. Shukla. Appl. Surf. Sci., 253:8889-8895, 2007.

[11]H. Zeng, W. Cai, Y. Li, J. Hu, and P. Liu. J. Phys. Chem. B, 109:18260-18266, 2005.

[12]S. Barcikowski, F. Devesa, and K. Moldenhauer. J. Nanopart. Res., 11:1883-1893, 2009.

[13]S. Barcikowski and F. Mafuné. J. Phys. Chem. C, 115(12):4985-5180, 2011.

[14]S. Barcikowski and F. Mafuné. J. Phys. Chem. C, 115:4985-4985, 2011.

[15]P. Wagener, G. Brandes, A. Schwenke, and S. Barcikowski. Phys. Chem. Chem. Phys., 13:5120-5126, 2011.

[16]J. Jakobi, S. Petersen, A. Menéndez-Manjón, P. Wagener, and S. Barcikowski. Langmuir, 26:6892$6897,2010$.

[17]N.G. Semaltianos, S. Logothetidis, N. Hastas, W. Perrie, S. Romani, R.J. Potter, G. Dearden, K.G. Watkins, P. French, and M. Sharp. Chem. Phys. Lett., 484:283-289, 2010.

[18]A. Hahn and S. Barcikowski. J. Laser Micro/Nanoeng., 4:51-54, 2009.

[19]V. Amendola and M. Meneghetti. Phys. Chem. Chem. Phys., 11(20):3805-3821, 2009.

[20]H.Y. Kwong, M.H. Wong, C.W. Leung, Y.W. Wong, and K.H. Wong. J. Appl. Phys., 108:034304, 2010.

[21]V. Amendola, P. Riello, and M. Meneghetti. J. Phys. Chem. C, 115:5140-5416, 2011.

[22]S. Besner, A.V. Kabashin, F.M. Winnik, and M. Meunier. J. Phys. Chem. C, 113:9526-9531, 2009.
[23]Q. Lu, S.S. Mao, X. Mao, and R.E. Russo. Appl. Phys. Lett., 80:3072-3074, 2002.

[24]S. Barcikowski, A. Menéndez-Manjón, B. Chichkov, M. Brikas, and G. Raciukaitis. Appl. Phys. Lett., 91:083113, 2007.

[25]P. Wagener, A. Schwenke, B.N. Chichkov, and S. Barcikowski. J. Phys. Chem. C, 114:7618-7625, 2010.

[26]P. Wagener, S. Faramarzi, A. Schwenke, R. Rosenfeld, and S. Barcikowski. Appl. Surf. Sci., 257:7231-7237, 2011.

[27]P.C. Painter, B.A. Brozoski, and M.M. Coleman. J. Polym. Sci. Polym. Phys. Ed., 20:1069-1080, 1982.

[28]B.A. Brozoski, M.M. Coleman, and P.C. Painter. Macromol., 17:230-234, 1984.

[29]D. Amans, C. Malaterre, M. Diouf, C. Mancini, F. Chaput, G. Ledoux, G. Breton, Y. Guillin, C. Dujardin, K. Masenelli-Varlot, and P. Perriat. J. Phys. Chem. C, 115:5131-5139, 2011.

[30]E. Giorgetti, F. Giammanco, P. Marsili, and A. Giusti. J. Phys. Chem. C, 115:5011-5020, 2011.

[31]Y. He, K. Kashiwagi, J. Fukuchi, K. Terao, A. Shirahata, and K. Igarashi. Eur. J. Biochem., 217:89-96, 1993.

[32]V.E. Orel, N.N. Dzyatkovskaya, A.V. Romanov, and T.M. Kozarenko. Exp. Oncol., 29:156-158, 2007.

[33]M.H. Oh, N. Lee, H. Kim, S.P. Park, Y. Piao, J. Lee, S.W. Jun, W.K. Moon, S.H. Choi, and T. Hyeon. J. Am. Chem. Soc., 133:5508-5515, 2011.

[34]A. Lifshitz, M. Bidani, and S. Bidani. J. Phys. Chem., 90:3422-3429, 1986.

[35]S. Barcikowski, A. Hahn, M. Guggenheim, K. Reimers, and A. Ostendorf. J. Nanopart. Res., 12:1733-1742, 2010.

[36]A. Menéndez-Manjón, P. Wagener, and S. Barcikowski. J. Phys. Chem. C, 115:5108-5114, 2011.

(Received: June 3, 2011, Accepted: December 14, 2011) 